\title{
The biology of ageing and the omics revolution
}

\author{
Lorna Harries • Katarzyna Goljanek-Whysall
}

Received: 18 September 2018/ Accepted: 25 September 2018/Published online: 4 October 2018

(C) Springer Nature B.V. 2018

Ageing is a multifactorial process associated with multiple mechanisms, some of which include telomere shortening, changes in metabolism associated with disrupted mitochondrial dynamics, stem cell exhaustion and cellular senescence or inflammation. Despite differences in physiological changes between different tissues, a set of unifying hallmarks of ageing has been defined providing a framework for understanding the process of ageing at cellular and organismal level. Given the complexity of the ageing process, the underlying mechanisms are still not well understood. The advent of-omics level technologies is now allowing a window on the molecular etiology of the ageing process, and how it contributes to age-related disease. New high throughput deep reach technologies now exist to query changes in ageing cells and populations at the level of the epigenome, the transcriptome, the proteome, the metabolome and the microbiome. These approaches are suitable for both hypothesis free discovery science, but also for detailed molecular characterization of specific phenotypes. Integration of these datasets represents a

L. Harries ( $\square)$

University of Exeter Medical School, Exeter, UK

e-mail: L.W.Harries@exeter.ac.uk

K. Goljanek-Whysall

Institute of Ageing and Chronic Disease, University of

Liverpool, Liverpool, UK

e-mail: k.whysall@liverpool.ac.uk challenge but also an opportunity for reaching a deeper understanding of the molecular causes and consequences of ageing.

In this special issue, Frenk and Houseley (2018) discuss the challenges of investigating molecular basis of ageing. The authors identify gene expression hallmarks of ageing and ask: is ageing a programmed process, is it conserved among species, do different tissues age at the same rate? Continuing the topic of biomarkers of ageing, the review by Pawelec (2017) offers an insight into a set of parameters characterizing ageing: "immune Risk Profile", IRP, a cluster of simple baseline immune parameters associated with excess mortality in older people, and discusses how IRPs are context-dependent. On the other hand, "immunosenescence", can be itself considered a hallmark of ageing. Ageing-associated remodelling of the immune system has significant impact on health of older people. Duggal (2018) summarise our current understanding of immunesenescence and discuss lifestyle and pharmacological interventions shown to restore immune functioning in aged individuals. Lorenzo et al. (2018), offer further insight into the consequences of ageing on immune system, focusing on $\mathrm{CD} 4+\mathrm{T}$ cell differentiation and function, linked to susceptibility of older people to infections, such as influenza.

Cellular senescence, not restricted to immunosenescence, is considered one of the key mechanisms 
underlying ageing. Accumulating senescent cells can contribute to disease progression not only through their changed function but also through affecting the surrounding environment. Burton and Faragher (2018) examine the underlying conceptual mechanisms by which senescent cells could contribute to a tissue functional decline and elegantly demonstrate that senescent cells are more than "simply irreversible proliferative arrest". The authors further discuss therapeutic targeting senescent cells for delaying age-related diseases, such as type-2 diabetes or metabolic syndrome. Lopez-Llunch (2018) further explores age-related diseases by focusing on mitochondrial dynamics. Mitochondria, the cells' powerhouses, are key organelles associated with metabolism and "mitochondrial theory" describes the efficiency of mitochondrial activity as key for longevity and ageing. Lopez-Lunch discusses interventions, such as caloric restriction, aiming at maintenance of bioenergetics homeostasis to delay ageing and prevent age-related diseases, such as sarcopenia. The current studies on mechanisms of sarcopenia and potential interventions to prevent or delay age-related loss of muscle mass and function, with focus on personalized approaches in conjunction with pharmacological interventions are further described in this issue by McCormick and Vasilaki (2018).

Changes in the number and/or functionality of stem cells have also been shown to contribute to age-related disorders. Adult stem cells are also used to target many changes that negatively impact tissue fitness with age. Rogers et al. (2018) discuss the role of circadian clocks in adult stem cell maintenance and how they control stem cell function. The topic of therapeutics is continued by Bikkul's article which focuses on a rare premature ageing disease: Hutchinson-Gilford Progeria Syndrome (HGPS) and drug treatments and regimes currently used in clinical trials (Bikkul et al. 2018). Finally, the review by Ezcurra (2018) discusses a new exciting research area in the field of ageing: how the human microbiome can change in specific disease states. However, it remains to be established if there is a causal link between microbial variation, disease and ageing.

In summary, this special issue brings together stateof-the-art thinking on the molecular causes and consequences of ageing, which may herald the development of an entirely new generation of anti-degenerative therapies. These may encompass modifiers of chromatin biology, regulators of mRNA processing, interventions to moderate mitochondrial number or function, interventions to address or repair DNA damage or tools to influence the metabolome, the proteome or the microbiome. Huge increases in our molecular toolkit have allowed insights into ageing processes that were previously impossible. We now face a challenge in translating these findings into meaningful advances in our understanding of the ageing process, and in translating them to the clinic.

\section{References}

Bikkul MU, Clements CS, Godwin LS, Goldberg MW, Kill IR, Bridger JM (2018) Farnelsyl transferase inhibitor and rapamycin correct aberrant genome organization and DNA damage in Hutchinson-Gilford Progeria Syndrome fibroblasts. Biogerontology. https://doi.org/10.1007/ s10522-018-9758-4

Burton D, Faragher R (2018) Obesity and type-2 diabetes as inducers of premature cellular senescence and ageing. Biogerontology. https://doi.org/10.1007/s10522-0189763-7

Duggal NA (2018) Reversing the immune ageing clock: lifestyle modifications and pharmacological interventions. Biogerontology. https://doi.org/10.1007/s10522-0189771-7

Ezcurra M (2018) Dissecting cause and effect in host-microbiome interactions using the combined worm- bug model system. Biogerontology. https://doi.org/10.1007/s10522018-9752-x

Frenk S, Houseley J (2018) Gene expression hallmarks of cellular ageing. Biogerontology. https://doi.org/10.1007/ s10522-018-9750-Z

Lopez-Llunch G (2018) Mitochondrial function in ageing and metabolism: shared mechanisms, shared outcomes? Biogerontology. https://doi.org/10.1007/s10522-0189768-2

Lorenzo EC, Bartley JM, Haynes L (2018) The impact of aging on $\mathrm{CD} 4+\mathrm{T}$ cell responses to influenza infection. Biogerontology. https://doi.org/10.1007/s10522-0189754-8

McCormick R, Vasilaki A (2018) Age-related changes in skeletal muscle: changes to life-style as a therapy. Biogerontology. https://doi.org/10.1007/s10522-0189775-3

Pawelec G (2017) Immune parameters associated with mortality in the elderly are context-dependent: lessons from Sweden, Holland and Belgium. Biogerontology. https://doi.org/10. 1007/s10522-017-9739-z

Rogers EH, Hunt JA, Pekovic-Vaughan V (2018) Adult stem cell maintenance and tissue regeneration around the clock: do impaired stem cell clocks drive age-associated tissue degeneration? Biogerontology. https://doi.org/10.1007/ s10522-018-9772-6 